\title{
Genetic architecture of fatty acid composition in the longissimus dorsi muscle revealed by genome-wide association studies on diverse pig populations
}

Wanchang Zhang ${ }^{\dagger}$, Junjie Zhang ${ }^{\dagger}$, Leilei Cui, Junwu Ma, Congying Chen, Huashui Ai, Xianhua Xie, Lin Li, Shijun Xiao, Lusheng Huang, Jun Ren ${ }^{*}$ and Bin Yang ${ }^{*}$ (D)

\begin{abstract}
Background: Fatty acid composition in muscle is an important factor that affects the nutritive value and taste of pork. To investigate the genetic architecture of fatty acid composition of pork, we measured fatty acid contents in longissimus dorsi muscle of 1244 pigs from three divergent populations and conducted genome-wide association studies (GWAS) for fatty acid contents.

Results: We detected 26 genome-wide significant quantitative trait loci (QTL) on eight chromosomes (SSC for Sus scrofa) for eight fatty acids. These loci not only replicated previously reported QTL for C18:0 on SSC14 and C20:0 on SSC16, but also included several novel QTL such as those for C20:1 on SSC7, C14:0 on SSC9, and C14:0, C16:0 and $\mathrm{C16:1}$ on SSC12. Furthermore, we performed a meta-analysis of GWAS on five populations, including the three populations that were investigated in this study and two additional populations that we had previously examined. This enhanced the strength of the associations detected between fatty acid composition and several marker loci, especially for those for C18:0 on SSC14 and C20:0 on SSC16. The genes ELOVL5, ELOVL6, ELOVL7, FASN, SCD and THRSP, which have functions that are directly relevant to fatty acid metabolism, are proximal to the top associated markers at six significant QTL.
\end{abstract}

Conclusions: The findings improve our understanding of the genetic architecture of fatty acid composition in pork and contribute to further fine-map and characterize genes that influence fatty acid composition.

\section{Background}

Pig meat represents about $40 \%$ of the red meat consumed by humans [1]. Fatty acids are essential cellular components and a major source of energy for animals. Fatty acid composition is closely related to the nutritive value and the taste of meat. Saturated fatty acids such as C14:0 and $\mathrm{C} 16: 0$ are risk factors for cardiovascular diseases such as coronary heart disease and atherosclerosis in humans [2]. In contrast, unsaturated fatty acids, especially omega-3

\footnotetext{
*Correspondence: renjunjxau@hotmail.com; binyang@live.cn 'Wanchang Zhang and Junjie Zhang are First co-authors State Key Laboratory for Pig Genetic Improvement and Production Technology, Jiangxi Agricultural University, Nanchang 330045, China
}

fatty acids, are beneficial to human health [3]. Identifying genomic regions associated with fatty acid composition in muscle would enable us to develop molecular breeding technologies to improve pork quality. In humans, fatty acid composition in muscle has been associated with insulin resistance [4]. Since the pig represents an important biomedical model for human diseases [5], dissection of the genetic architecture of fatty acid composition in pork can also provide insights into the molecular mechanisms that underlie fatty acid metabolism in humans.

Genome-wide association studies (GWAS) have been performed to identify genomic regions associated with a variety of traits in pigs [6-8] and significant quantitative trait loci (QTL) that influence fatty acid composition 
have been identified in several pig populations. These QTL harbor strong candidate genes such as SCD [9], ELOVL6 [10] and ELOVL7 [9]. However, the genetic basis that underlies variation in fatty acid composition is still largely unclear. For instance, further investigations are needed to reveal additional QTL that affect fatty acid composition in pork from diverse breeds. Moreover, a meta-analysis of results from multiple populations is required to improve the detection power of GWAS for fatty acid composition in pork.

In our previous study, we reported GWAS on fatty acid composition in longissimus dorsi muscle samples from a White Duroc $\times$ Erhualian $F_{2}$ intercross (hereafter referred to as $\left.\mathrm{F}_{2}, \mathrm{n}=591\right)$ and Sutai pigs $(\mathrm{n}=282)$ [9]. Here, we recorded fatty acid contents in the longissimus dorsi muscle and obtained $\sim 60 \mathrm{~K}$ single nucleotide polymorphism (SNP) genotypes on 1244 pigs from three additional populations: two Chinese indigenous breeds (Laiwu, $\mathrm{n}=305$; Erhualian, $\mathrm{n}=331$ ) and a Duroc $\times$ (Landrace $\times$ Yorksire) (DLY) three-way hybrid commercial population $(n=608)$. We performed GWAS on each of these three populations and identified a list of novel significant QTL that harbor interesting candidate genes. We also conducted a meta-analysis of GWAS on five populations, including the three populations (Laiwu, Erhualian and DLY) that we tested in this study and the two populations $\left(\mathrm{F}_{2}\right.$ and Sutai) that we previously investigated. The large sample size and broad genetic background of our tested populations allowed us not only to confirm previously reported QTL, but also to identify novel QTL and candidate genes.

\section{Methods}

\section{Animals}

Five pig populations were used in this study, including Laiwu, Erhualian, DLY, $\mathrm{F}_{2}$, and Sutai pigs. Laiwu is a Chinese indigenous pig breed that was originally distributed in Laiwu, Shangdong province. This breed is known for its unusually high intramuscular fat content (9-12\%) [11]. Erhualian pigs are mainly located in Wuxi, Jiangsu Province, and are famous for their large litter size [11]. We purchased 385 Laiwu and 390 Erhualian piglets at the age of 90 days from nucleus farms of these two breeds in Shandong and Jiangsu provinces, respectively. These pigs were selected to represent offspring of all boars and the majority of sows in these two farms. The Laiwu pigs were derived from 25 sires and 115 dams and the Erhualian pigs were offspring of 11 sires and 55 dams. These animals were transferred to and raised in a pig farm in Nanchang, Jiangxi Province. Boars were castrated before day 90. All Erhualian and Laiwu pigs were fed a corn-soybean based diet containing $16 \%$ crude protein, $3100 \mathrm{~kJ}$ digestible energy and $0.78 \%$ lysine under standard management conditions. A total of 333 Laiwu and 336 Erhualian pigs were uniformly slaughtered at $300 \pm 3$ days in 18 and 11 batches, respectively. A total of 698 DLY pigs were purchased from a commercial pig farm in Xiushui, Jiangxi Province. The DLY boars were castrated at day 25 and all pigs were fed a corn-soybean diet containing $16 \%$ crude protein, $3132 \mathrm{~kJ}$ digestible energy and $0.85 \%$ lysine, and were slaughtered at $180 \pm 3$ days in 22 batches.

To conduct a meta-analysis of GWAS, we also used the $60 \mathrm{~K} \mathrm{SNP}$ genotypes and fatty acid composition phenotypes from two other populations: $\mathrm{F}_{2}$ and Sutai pigs. The pedigree, management and genotype information of these two populations were described previously [9]. In brief, the $\mathrm{F}_{2}$ population comprised $1912 \mathrm{~F}_{2}$ pigs that were derived from two White Duroc founder boars and 17 Chinese Erhualian founder sows. The Sutai population is a Chinese synthetic pig line that originated from a cross between Chinese Taihu and European Duroc pigs and then artificially selected for more than 18 generations [11]. Both $F_{2}$ and Sutai pigs were fed with the same diet that was used for the Erhualian and Laiwu pigs during the fattening period.

\section{Ethics statements}

All the experiments that involved animals were carried out in accordance with the approved guidelines by the Ministry of Agriculture of China. Approval was obtained from the ethics committee of Jiangxi Agricultural University before the experiment.

\section{Phenotype recording}

Approximately $50 \mathrm{~g}$ of longissimus dorsi tissue was dissected from the 3rd to 4th lumbar vertebrae region of each pig and frozen in liquid nitrogen within $30 \mathrm{~min}$ post-mortem, and then stored at $-80^{\circ} \mathrm{C}$ for further use. About $10 \mathrm{~g}$ of longissimus dorsi tissue was ground and then treated with a 3:1 chloroform-methanol solution according to [12]. Then, $2 \mathrm{mg}$ of extracted lipids was redissolved in $2 \mathrm{~mL}$ of $\mathrm{n}$-hexane and $1 \mathrm{~mL}$ of $\mathrm{KOH}(0.4 \mathrm{M})$ for saponification and methylation. Fatty acid methyl esters were processed with a GC2010 Plus Gas Chromatograph (Shimadzu), following manufacturer recommendations. Signals for each fatty acid were quantified relative to standard reference reagents (Sigma-Aldrich) and the percentage of each fatty acid relative to total fatty acids was used as phenotype for further analyses. The same measurement procedures were used for all five populations.

\section{Genotype data}

A standard phenol/chloroform method was used to extract genomic DNA from ear tissue of each pig. All animals were genotyped with Illumina Porcine SNP60 
BeadChips (v2) [13] according to the manufacturer's protocol. We used the same quality control procedures on genotype data for all tested populations. Briefly, we selected SNPs that had a call rate greater than 0.9 and a minor allele frequency (MAF) greater than 0.01 , and individuals that had a genotype call rate higher than 0.9, for further analyses. All quality control procedures were implemented by Plink v1.07 [14].

\section{Estimation of phenotypic correlations}

To estimate phenotypic correlations between different fatty acid contents, we adjusted the content of each fatty acid in each population separately by treating sex and slaughter batch as fixed effects and polygenic effects as random effects in a single-trait linear mixed model implemented by the polygenic function in the $\mathrm{R}$ package GenABEL [15]. The variance-covariance matrix of polygenic effects was estimated based on whole-genome SNP genotype data. Phenotypic correlations were measured as spearman correlation coefficients. Heatmaps of the correlation matrix were plotted by the "heatmap.2" function of the gplots package in $\mathrm{R}$ software (https://cran.r-project.org/web/packages/gplots/).

\section{Estimation of heritability and GWAS}

The proportion of phenotypic variance explained by whole-genome SNP genotypes i.e., heritability, was estimated using the $-1 \mathrm{~mm}$ procedure of GEMMA [16] based on the genomic relationship matrix. Sex and batch were included as fixed effects and the estimation was done separately for each population. For GWAS, we assessed the association between phenotypes and each SNP across the genome under the following linear mixed model:

$$
\mathbf{y}=\mathbf{X b}+\mathbf{Z a}+\mathbf{k g}+\mathbf{e},
$$

where $\mathrm{y}$ is a vector of phenotypic values, $\mathbf{X}, \mathbf{Z}$, and $\mathbf{k}$ are incidence matrices for fixed effects (sex and batch), polygenic effects and SNP genotypes, respectively. $\mathbf{Z}$ is an identity matrix, $\mathbf{k}$ is a vector of $0,1,2$ values, where 0 and 2 represent the two alternative homozygotes and 1 denotes the heterozygote at the evaluated SNP, $\mathbf{b}$ is a vector of fixed effects, $\mathbf{a}$ is a vector of polygenic effects with distribution: $\mathrm{N}\left(0, \mathbf{G} \sigma_{A}^{2}\right)$, with $\mathbf{G}$ the genomic relationship matrix based on genome-wide SNPs and $\sigma_{A}^{2}$ the additive genetic variance, $g$ is the additive genetic effect of the tested SNP, and $\mathbf{e}$ is a vector of residuals. We identified significant SNPs through a stepwise forward selection in multiple rounds of genome scans, where the most significant SNPs that were identified in previous genomic scans were included as additional fixed effects in the model for the next round of scan until no SNP passed the suggestive significant threshold [17]. The threshold for defining a suggestive significant $\mathrm{SNP}$ was set to $1 / \mathrm{N}_{\mathrm{snp}}$, where $\mathrm{N}_{\mathrm{snp}}$ was the number of SNPs tested in genome-wide scans. Genome-wide significance thresholds were set to 0.05 / $\mathrm{N}_{\text {snp }}$.

The GWAS meta-analysis was performed using the METAL program [18], which tests the combined effects and standard errors of effects of each common SNP across the tested populations by taking sample size and direction of genotype effects into account. The phenotypic variance explained by significant GWAS SNPs was estimated as $\left(\mathrm{V}_{\text {reduce- }} \mathrm{V}_{\text {full }}\right) / \mathrm{V}_{\text {reduce, }}$, where $\mathrm{V}_{\text {reduce }}$ and $\mathrm{V}_{\text {full }}$ are the residual variances of ordinary linear models with and without including SNP genotypes as predictor variables, respectively.

\section{Characterization of candidate genes}

To identify functionally plausible candidate genes near the genome-wide significant SNPs, we examined annotated genes around the region that was centered at each top SNP that was identified by GWAS in the Sus scrofa assembly (Build 10.2). Because annotation of the pig reference genome is far from perfect, we also investigated homologous segments in other mammalian species, including mouse, cattle and humans (http://genome.ucsc. edu). Among the multiple genes that were found within target regions, we prioritized candidate genes according to their physical distance to the top SNPs and their functional relevance to fatty acid metabolism.

\section{Results and discussion \\ Phenotypes}

We focused on 12 fatty acids that account for $~ 95 \%$ of the total fatty acids (Table 1). The most abundant fatty acid was C18:1, followed by $\mathrm{C} 16: 0$ and C18:0, across the populations analyzed. Heritability estimates for most fatty acids ranged from 0.4 to 0.8 (Table 1 ), which suggests that the contribution of genetic effects to phenotypic variation in fatty acid composition is considerable. We assessed phenotypic correlations between fatty acid contents for the Laiwu, Erhualian and DLY populations. Briefly, polyunsaturated fatty acids, including C18:2, C18:3, C20:2, C20:3 and C20:4, were positively correlated with each other. These fatty acids were clustered into a module that was distinct from the saturated and monounsaturated fatty acids (Fig. 1). Saturated fatty acids (C14:0, C16:0, C18:0 and C20:0) were also positively correlated with each other. These correlation patterns were conserved across the three populations (Fig. 1).

\section{GWAS in the Erhualian, Laiwu and DLY populations}

After quality control, 35,974 SNPs for 305 Erhualian pigs, 49,343 SNPs for 331 Laiwu pigs, and 56,216 SNPs for 608 DLY pigs were retained for GWAS. We discarded 24,893, 
Table 1 Summary statistics for fatty acid composition in three pig populations

\begin{tabular}{|c|c|c|c|c|c|c|c|c|c|}
\hline \multirow[t]{2}{*}{ Trait } & \multicolumn{3}{|c|}{ Laiwu } & \multicolumn{3}{|c|}{ Erhualian } & \multicolumn{3}{|l|}{ DLY } \\
\hline & $\mathrm{N}$ & Mean \pm SD & $h^{2} \pm S E$ & $\mathrm{~N}$ & Mean \pm SD & $h^{2} \pm S E$ & $\mathrm{~N}$ & Mean \pm SD & $h^{2} \pm \mathrm{SE}$ \\
\hline Myristic (C14:0) & 305 & $1.34 \pm 0.12$ & $0.67 \pm 0.09$ & 331 & $1.34 \pm 0.18$ & $0.81 \pm 0.08$ & 608 & $1.31 \pm 0.12$ & $0.51 \pm 0.08$ \\
\hline Palmitic (C16:0) & 305 & $26.50 \pm 1.00$ & $0.56 \pm 0.08$ & 331 & $25.00 \pm 1.30$ & $0.73 \pm 0.08$ & 608 & $23.88 \pm 1.22$ & $0.56 \pm 0.08$ \\
\hline Palmitoleic (C16:1n-7) & 305 & $3.87 \pm 0.60$ & $0.70 \pm 0.06$ & 331 & $3.62 \pm 0.68$ & $0.77 \pm 0.08$ & 608 & $3.91 \pm 0.46$ & $0.67 \pm 0.09$ \\
\hline Stearic (C18:0) & 305 & $12.09 \pm 1.11$ & $0.57 \pm 0.09$ & 331 & $12.58 \pm 1.25$ & $0.75 \pm 0.09$ & 608 & $11.36 \pm 1.14$ & $0.64 \pm 0.09$ \\
\hline Oleic (C18:1n-9) & 305 & $46.42 \pm 1.58$ & $0.48 \pm 0.10$ & 331 & $46.17 \pm 2.26$ & $0.76 \pm 0.09$ & 608 & $45.36 \pm 2.04$ & $0.46 \pm 0.09$ \\
\hline Linoleic (C18:2n-6) & 305 & $3.63 \pm 0.80$ & $0.01 \pm 0.05$ & 331 & $4.46 \pm 0.89$ & $0.48 \pm 0.10$ & 608 & $5.40 \pm 1.51$ & $0.29 \pm 0.09$ \\
\hline Linolenic (C18:3n-3) & 305 & $0.11 \pm 0.03$ & $0.12 \pm 0.09$ & 331 & $0.13 \pm 0.03$ & $0.45 \pm 0.10$ & 608 & $0.19 \pm 0.07$ & $0.26 \pm 0.09$ \\
\hline Arachidic (C20:0) & 305 & $0.19 \pm 0.03$ & $0.72 \pm 0.11$ & 331 & $0.25 \pm 0.05$ & $0.73 \pm 0.08$ & 608 & $0.19 \pm 0.03$ & $0.77 \pm 0.08$ \\
\hline Eicosenoic (C20:1n-9) & 305 & $0.75 \pm 0.15$ & $0.82 \pm 0.07$ & 331 & $0.74 \pm 0.13$ & $0.63 \pm 0.10$ & 608 & $0.86 \pm 0.11$ & $0.46 \pm 0.08$ \\
\hline Eicosadienoic (C20:2n-6) & 305 & $0.20 \pm 0.04$ & $0.18 \pm 0.10$ & 331 & $0.25 \pm 0.05$ & $0.59 \pm 0.09$ & 608 & $0.28 \pm 0.07$ & $0.43 \pm 0.09$ \\
\hline Homolonolenic (C20:3n-6) & 305 & $0.05 \pm 0.01$ & $0.26 \pm 0.10$ & 331 & $0.08 \pm 0.02$ & $0.39 \pm 0.10$ & 602 & $0.09 \pm 0.03$ & $0.18 \pm 0.08$ \\
\hline Arachidonic (C20:4n-6) & 305 & $0.18 \pm 0.08$ & $0.20 \pm 0.10$ & 331 & $0.25 \pm 0.09$ & $0.46 \pm 0.10$ & 608 & $0.48 \pm 0.29$ & $0.13 \pm 0.07$ \\
\hline
\end{tabular}

$\mathrm{N}$ stands for the number of individuals in each population

$h^{2}$ represents the heritability of each trait for each population; heritabilities were estimated using the -I mm procedure of GEMMA based on genome-wide SNP genotypes [16]

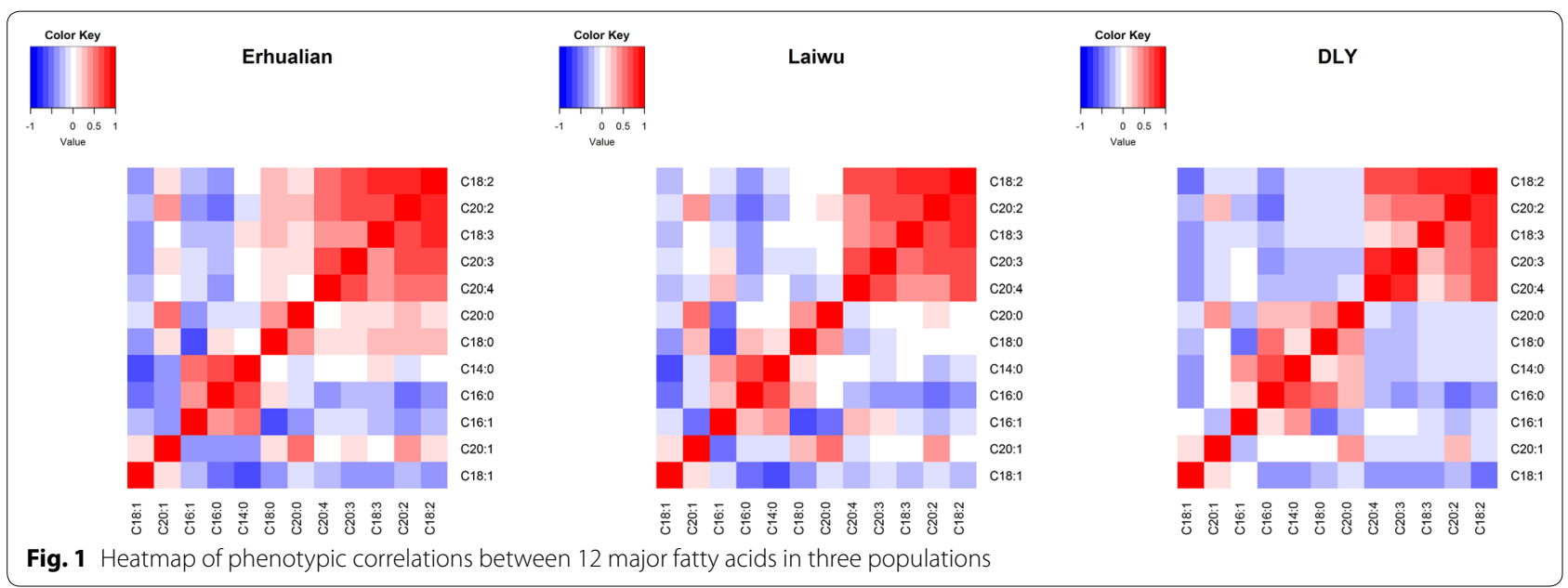

11,249 and 4806 SNPs with a MAF lower than 0.01 for the Erhualian, Laiwu and DLY populations, respectively. Different numbers of rare SNPs significantly resulted in different numbers of qualified SNPs in the three populations. This was as expected because SNPs on the porcine Illumina $60 \mathrm{~K}$ chips were selected mainly based on European pigs that have diverged from Chinese indigenous pigs [13]. Therefore, ascertainment bias was unavoidable, leading to a higher proportion of rare SNPs for the Chinese Laiwu and Erhualian pigs than for the DLY pigs.

We identified 26 genome-wide significant QTL on eight chromosomes for eight fatty acid contents (Fig. 2a; Table 2). These QTL explained from 3.3 to $35.2 \%$ of the phenotypic variance for a fatty acid phenotype (Table 2). The QTL for C18:0 content on SSC14 and C20:0 content on SSC16 replicated QTL that were previously reported for these traits in the $F_{2}$ and Sutai populations [9], and the QTL for C16:1 content on SSC8 overlapped with the QTL for this trait identified in an Iberian $\times$ Landrace intercross [7]. To our knowledge, the other QTL were detected for the first time, including the QTL for C20:1 around 134.54 Mb on SSC7, C14:0 around 13.83 Mb on SSC9, and C14:0 and C16:0 around 1.7 Mb on SSC12 (Table 2).

\section{Shared and specific QTL across populations}

We also conducted a meta-analysis of GWAS to assess the combined association signals for the five populations: $\mathrm{F}_{2}$, Sutai, Erhualian, Laiwu and DLY. The meta-analysis did not identify new QTL but enhanced the strength of 


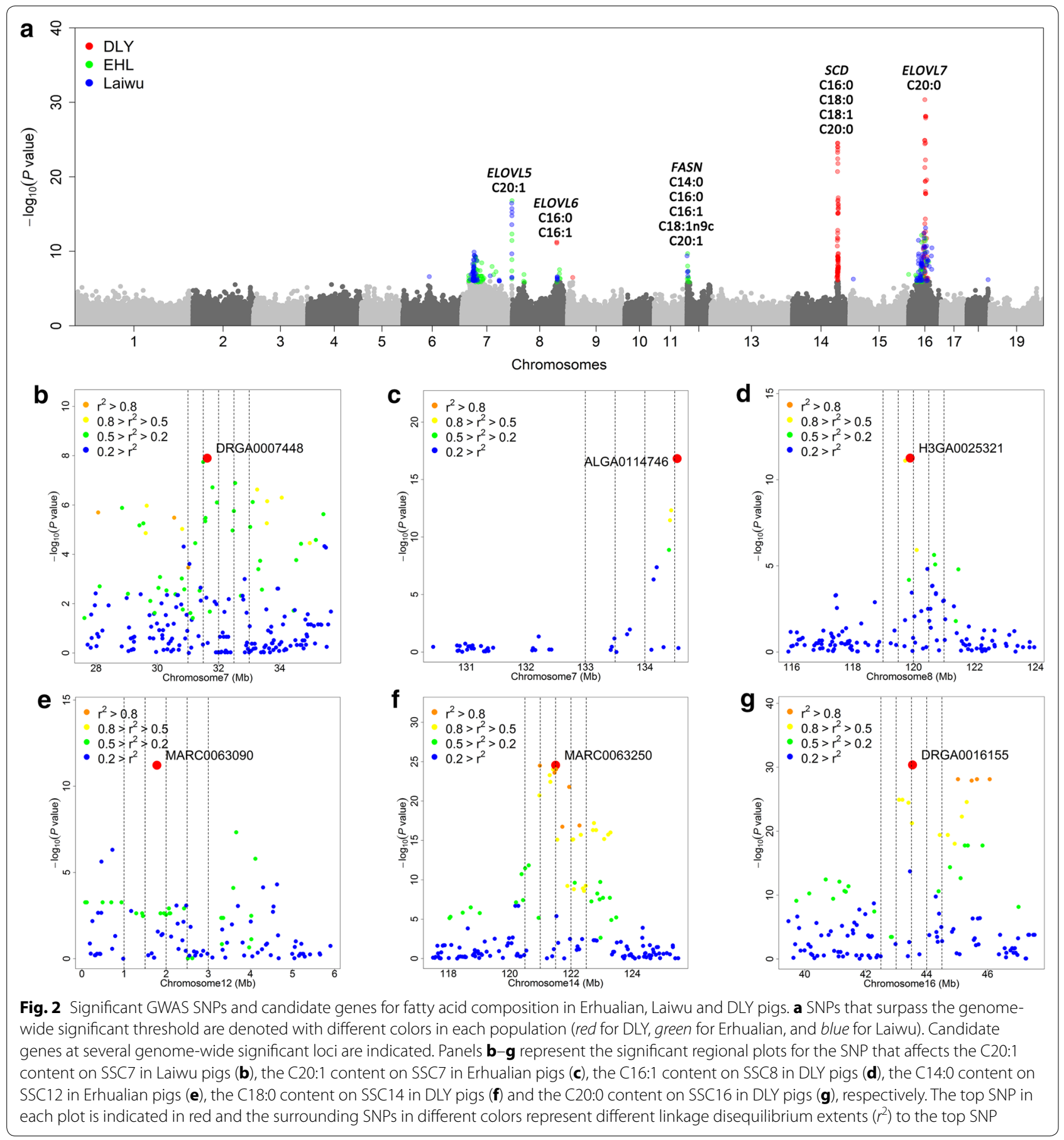

associations between fatty acid composition and several markers, especially for the QTL for C18:0 on SSC14 and C20:0 on SSC16 (see Additional file 1: Figure S1). The $P$ value of the top SNP (CASI0010164) for C18:0 increased from $2.82 \times 10^{-25}$ for DLY pigs to $5.06 \times 10^{-44}$ in the metaanalysis, and the $P$-value of the top SNP (DRGA0016155) for C20:0 on SSC16 increased from $4.32 \times 10^{-31}$ in DLY pigs to $3.85 \times 10^{-47}$ in the meta-analysis. The direction of SNP effects was concordant across the five populations, with allele $C$ at SNP CASI0010164 and allele $G$ at SNP DRGA0016155 increasing C18:0 and C20:0 contents, respectively. This indicates that common or closely-linked functional variants may be responsible for the GWAS signals in these populations. 
Table 2 Genome-wide significant QTL for fatty acid composition in Erhualian, Laiwu and DLY pig populations

\begin{tabular}{|c|c|c|c|c|c|c|c|c|c|}
\hline Chr & Trait & Pop & Nsnp & Range of Nsnp (Mb) & Top SNP & Position (bp) & $P$-value & $\operatorname{Var}(\%)$ & Candidates \\
\hline 6 & $\mathrm{C} 16: 1$ & Laiwu & 1 & 71.01 & ASGA0106005 & 71011913 & $1.97 \mathrm{E}-07$ & 4.95 & \\
\hline 7 & C20:1 & Laiwu & 18 & $27.35-31.80$ & H3GA0020505 & 29416373 & $2.25 \mathrm{E}-08$ & 7.91 & \\
\hline 7 & $\mathrm{C} 14: 0$ & Laiwu & 15 & $29.54-33.59$ & ALGA0039950 & 31500144 & $2.60 \mathrm{E}-08$ & 17.78 & \\
\hline 7 & C16:1 & Laiwu & 21 & $29.42-34.06$ & DRGA0007448 & 31628039 & $1.27 \mathrm{E}-08$ & 8.03 & \\
\hline 7 & C20:2n6 & Laiwu & 6 & $29.42-34.06$ & DRGA0007448 & 31628039 & $5.61 \mathrm{E}-07$ & 6.55 & \\
\hline 7 & C20:1 & Laiwu & 9 & $133.96-134.54$ & ASGA0037322 & 133962789 & $3.54 \mathrm{E}-17$ & 35.18 & ELOVL5 \\
\hline 7 & C20:1 & Erhualian & 6 & $134.15-134.54$ & ALGA0114746 & 134540651 & $1.51 \mathrm{E}-17$ & 26.43 & \\
\hline 8 & C16:1 & DLY & 7 & $119.73-121.47$ & H3GA0025321 & 119887525 & $1.54 \mathrm{E}-13$ & 8.31 & ELOVL6 \\
\hline 8 & $\mathrm{C} 16: 0$ & Laiwu & 20 & $118.82-123.41$ & ALGA0049254 & 120996107 & $2.66 \mathrm{E}-07$ & 8.89 & \\
\hline 8 & $\mathrm{C} 16: 1$ & Erhualian & 11 & $125.08-129.51$ & ALGA0049349 & 127072110 & $6.18 \mathrm{E}-09$ & 9.77 & \\
\hline 9 & C20:1 & DLY & 4 & $6.29-7.58$ & ALGA0105252 & 6304559 & $4.27 \mathrm{E}-07$ & 3.99 & \\
\hline 9 & $\mathrm{C} 14: 0$ & DLY & 4 & $13.26-14.31$ & ALGA0107040 & 13826057 & $2.15 E-08$ & 3.33 & THRSP \\
\hline 9 & $\mathrm{C} 14: 0$ & DLY & 4 & $13.26-14.31$ & MARC0100725 & 14217867 & $3.23 \mathrm{E}-07$ & 4.00 & \\
\hline 12 & C14:0 & Laiwu & 6 & $0.25-2.52$ & ASGA0099260 & 248014 & $4.06 \mathrm{E}-10$ & 9.01 & \\
\hline 12 & C14:0 & Erhualian & 2 & $0.46-4.12$ & MARC0063090 & 1779278 & $6.05 E-12$ & 26.81 & FASN \\
\hline 12 & C16:0 & Erhualian & 3 & $0.46-4.12$ & MARC0063090 & 1779278 & $9.24 \mathrm{E}-09$ & 19.56 & \\
\hline 12 & C16:1 & Erhualian & 4 & $0.46-4.12$ & MARC0063090 & 1779278 & $2.01 \mathrm{E}-10$ & 13.07 & \\
\hline 12 & C18:1n9c & Erhualian & 4 & $0.46-4.12$ & MARC0063090 & 1779278 & $1.14 \mathrm{E}-06$ & 17.44 & \\
\hline 12 & C20:1 & Erhualian & 5 & $0.46-4.12$ & MARC0063090 & 1779278 & $1.03 \mathrm{E}-07$ & 10.97 & \\
\hline 14 & $\mathrm{C} 16: 0$ & DLY & 11 & $120.39-121.95$ & ALGA0081087 & 120972588 & $4.00 \mathrm{E}-08$ & 6.25 & $S C D$ \\
\hline 14 & $\mathrm{C} 20: 0$ & DLY & 4 & $120.97-121.50$ & CASI0010164 & 121305916 & $1.04 \mathrm{E}-07$ & 3.51 & \\
\hline 14 & C18:0 & DLY & 45 & $119.03-123.48$ & MARC0063250 & 121500518 & $2.82 \mathrm{E}-25$ & 26.35 & \\
\hline 14 & C16:1 & DLY & 35 & $120.51-123.29$ & ASGA0066120 & 121515129 & $1.47 \mathrm{E}-13$ & 11.13 & \\
\hline 16 & $C 20: 0$ & Erhualian & 20 & $32.43-36.85$ & ASGA0072949 & 34715842 & $6.97 \mathrm{E}-13$ & 12.07 & \\
\hline 16 & $\mathrm{C} 20: 0$ & DLY & 37 & $41.14-45.83$ & DRGA0016155 & 43534471 & $4.32 \mathrm{E}-31$ & 31.79 & ELOVLT \\
\hline 16 & C20:0 & Laiwu & 4 & $43.53-45.31$ & DRGA0016169 & 45313348 & $7.41 \mathrm{E}-14$ & 25.50 & \\
\hline
\end{tabular}

$P$-values were calculated using the GenABEL package in $\mathrm{R}$

Phenotypic variation explained by the top SNPs was estimated by $\left(V_{\text {reduce }}-V_{\text {full }}\right) / V_{\text {resuce, }}$ where $V_{\text {reduce }}$ and $V_{\text {full }}$ are the residual variances of models with and without including SNP genotypes as predictor variables, respectively

Chr chromosome, Pop population, $N_{\text {snp }}$ number of significant SNPs, Position genomic position on the Sscrofa 10.2 pig genome assembly, Var (\%) percentage of phenotypic variance explained by each locus

We identified several genomic regions that showed genome-wide significant association signals in multiple populations, including the regions between 134.0 and 134.5 Mb on SSC7 for C20:1, between 120.0 and 127.1 Mb on SSC8 for C16:1, and between 0.21 and $1.87 \mathrm{Mb}$ on SSC12 for C14:0 (Table 2). The extent of linkage disequilibrium (LD) is usually small in diverse populations. For example, LD at $r^{2}=0.3$ extends across 10.5 and $125 \mathrm{~kb}$ in Chinese and European pigs, respectively [19]. The $60 \mathrm{~K}$ SNPs on the Illumina chip are not likely the causative mutations for the observed associations. However, it is reasonable to hypothesize that the population-shared signals observed here arise from common causative mutations. The top SNPs for multiple associations across diverse populations must be in the vicinity of the causative mutations, providing insights to further characterize these causative mutations.

\section{Candidate genes for genome-wide significant QTL}

In most mammals, fatty acids up to palmitate (C16:0) are synthesized de novo from malonyl-CoA and acetyl-CoA by fatty acid synthetases (FAS) in the liver and fat tissues [20]. However, some fatty acids (essential fatty acids), such as linoleic acids (C18:2n-6) and linolenic acids $(\mathrm{C} 18: 3 n-3)$, are normally found in vegetables and nuts and cannot be de novo synthesized in mammals. Within cells, fatty acids can be metabolized or transformed by different elongases and desaturases. For example, SCD (stearoyl-CoA desaturase) desaturates the saturated fatty acids $\mathrm{C} 16: 0$ and $\mathrm{C} 18: 0$ to the mono-unsaturated fatty acids C16:1n-7 and C18:1n-9. Different elongases (ELOVL2 and ELOVL5) and desaturases ( $\Delta 5$ and $\Delta 6$ desaturases) are involved in catalyzing $n-3$ and $n-6$ polyunsaturated fatty acids [20]. Therefore, it is conceivable that altered enzymatic activities of these synthetases, elongases and desaturases affect fatty acid composition in 
cells or tissues. This contributes fundamental knowledge to characterize candidate genes for genome-wide significant QTL.

On SSC7, we detected two significantly associated regions in the genome of the Laiwu and Erhualian pigs but not of DLY pigs (Table 2; Fig. 2). The region around $31.63 \mathrm{Mb}$ was associated with $\mathrm{C} 14: 0$ and $\mathrm{C} 16: 1$ contents in Laiwu pigs (Fig. 2b). The other region, around 134.54 Mb, was associated with the C20:1 content in both Erhualian and Laiwu pigs. The top SNP ALGA0114746 for the $134.54 \mathrm{Mb}$ region in the Erhualian pigs is located at about $5 \mathrm{~kb}$ upstream of the ELOVL5 (elongation of very long chain fatty acid protein 5) gene (between 134.54 and $134.62 \mathrm{Mb}$ ) (Fig. 2c). ELOVL5 encodes an elongase that plays an important role in determining de novo synthesis of monounsaturated fatty acid in mice [21]. Therefore, ELOVL5 is a strong candidate for this QTL that affects C20:1 content.

On SSC8, we found significant signals for C16:0 and C16:1 contents in Erhualian, Laiwu and DLY pigs (Table 2). The most significantly associated SNP $\left(P=1.54 \times 10^{-13}\right)$ was H3GA0025321 at $119.88 \mathrm{Mb}$ for $\mathrm{C} 16: 1$ for DLY pigs (Fig. 2d). The top SNP (ALGA0049254) for C16:0 for Laiwu pigs was at $121.00 \mathrm{Mb}$. This region (between 119.88 and $121.00 \mathrm{Mb}$ ) overlapped with the QTL (between 117.82 and $120.10 \mathrm{Mb}$ ) for C16:1 that was identified for Iberian $\times$ Landrace $F_{2}$ and backcross populations [7, 22]. This region contains the ELOVL6 (elongation of very long chain fatty acid protein 6) gene at $120.12 \mathrm{Mb}$. ELOVL6 plays a key role in catalyzing elongation of 16-18 carbon fatty acids [23]. The level of expression of this gene has been associated with $\mathrm{C} 16: 0$ and $\mathrm{C} 16: 1$ contents in pig muscle [10]. Interestingly, allele $C$ at the top SNP (H3GA0025321) was associated with decreased C16:1 content and increased C18:1 content $\left(P=8.9 \times 10^{-5}\right)$, which is consistent with the enzymatic activity of ELOVL6. Similar scenarios were also found for the Erhualian and Laiwu pigs.

At 13.82 and $14.21 \mathrm{Mb}$ on SSC9, we found SNPs that were significantly associated with C14:0 content in DLY pigs but not in Erhualian or Laiwu pigs (Table 2). The top SNP at $13.82 \mathrm{Mb}$ was located in an intron of the NDUFC2 (NADH dehydrogenase 1 subunit $C 2$ ) gene, which has no apparent functional relevance to fatty acid metabolism. We further investigated homologous regions in other species, including humans and mice, and found that the region that is homologous to the segment between 13.81 and $13.82 \mathrm{Mb}$ on SSC9 contains the THRSP (thyroid hormone responsive) gene. THRSP is involved in the regulation of genes that are involved in fatty acid synthesis [24]. Interestingly, the homologous region in the bovine genome is associated with muscle fatty acid composition in cattle [25]. Therefore, we consider THRSP as an interesting candidate gene that warrants further investigation.

On SSC12, we identified significant SNPs for C14:0 content in the region between 0.21 and $1.87 \mathrm{Mb}$ in both Erhualian and Laiwu pigs (Table 2). The top SNP (ASGA0099260, $P=4.06 \times 10^{-10}$ ) detected for the Laiwu pigs was located at $0.25 \mathrm{Mb}$, which is about $1.52 \mathrm{Mb}$ away from the top SNP (MARC0063090, $P=6.05 \times 10^{-12}$ ) at $1.77 \mathrm{Mb}$ that was identified for Erhualian pigs (Fig. 2e). Allele $A$ at the top SNP for Erhualian pigs was significantly associated with increased C14:0, C16:0 and C16:1 contents but with decreased C18:1 and C20:1 contents (Table 2). The same association pattern was found at this QTL for Laiwu pigs, although $P$ values of the top SNPs for $\mathrm{C} 16: 0\left(P=4.3 \times 10^{-4}\right), \mathrm{C} 16: 1\left(P=1.7 \times 10^{-4}\right)$, C18:1 $\left(P=4.7 \times 10^{-5}\right)$ and C20:1 $\left(P=2.6 \times 10^{-4}\right)$ did not reach the genome-wide significance threshold. For DLY pigs, although ASGA0099260 (MAF $=0.23)$ and MARC0063090 (MAF $=0.42)$ had high MAF, no significant association was found. This indicates that causal variant(s) at this QTL may have a population-specific effect in Erhualian and Laiwu pigs. Another possible reason is that there is no genetic variation at the causal variant in DLY pigs. At $1.02 \mathrm{Mb}$ on this chromosome, we found the FASN (fatty acid synthase) gene, which encodes an enzyme involved in the synthesis of C16:0 from acetylCoA and malonyl-CoA [26].

On SSC14, SNPs within a $\sim 500 \mathrm{~kb}$ region (between 120.97 and $121.50 \mathrm{Mb}$ ) were significantly associated with C16:0, C16:1, C18:0 and C20:0 contents in DLY but not in Erhualian and Laiwu pigs (Table 2). Within this region, the most significant association was observed between MARC0063250 at $121.50 \mathrm{Mb}$ and $\mathrm{C} 18: 0$ content in DLY pigs $\left(P=2.82 \times 10^{-25}\right)$ (Fig. 2f). The top SNP explained $26.4 \%$ of phenotypic variance. It should be noted that this region overlapped with a previously reported QTL for C18:0 in the $\mathrm{F}_{2}$ and Sutai populations [9]. The $S C D$ gene at $120.96 \mathrm{Mb}$ was proposed as a strong candidate gene for this QTL. SCD encodes a rate-limiting enzyme that preferably catalyzes palmitoyl-(C16:0) and stearoyl-(C18:0) to palmitoleoyl-CoA (C16:1) and oleoyl-CoA (C18:1) [27]. SCD polymorphisms have been associated with fatty acid composition in pig, cattle and sheep [28-30]. Interestingly, in this study, allele $A$ at the top SNP (MARC0063250) showed positive effects on C16:0 and C18:0 contents but negative effects on C16:1 and C18:1 contents (Table 2), which agrees with the enzymatic activity of SCD.

It should be noted that we identified a significant QTL for C20:0 around 43.51 Mb on SSC16 in Erhualian, Laiwu and DLY pigs, despite the divergent genetic background between Chinese indigenous and DLY pigs. This region also showed significant association signals for C20:0 in 
the $\mathrm{F}_{2}$ and Sutai pigs. In this study, the most significant associated SNP (DRGA0016155, $P=4.32 \times 10^{-31}$ ) was identified for DLY pigs at $43.51 \mathrm{Mb}$ on this chromosome. This SNP was also the top SNP $\left(P=3.85 \times 10^{-47}\right)$ in the meta-analysis. However, the top SNPs differed between the five populations, with distances of 1 to $10 \mathrm{Mb}$ between each other. One explanation is that the marker density of the current $60 \mathrm{~K} \mathrm{SNP}$ array is still not sufficient to capture the across-population LD between causative variants and SNPs. Another explanation could be that the causative variants differ between populations. In our previous study, we proposed ELOVL7 (elongation of very long chain fatty acid protein 7) at $42.50 \mathrm{Mb}$ as an interesting candidate gene for the SSC16 locus, since $E L O V L 7$ encodes an elongase that is directly involved in the metabolism of $\mathrm{C} 20: 0$ and is about $200 \mathrm{~kb}$ away from the strongest SNP that was found for Sutai pigs. We note that ELOVL7 is $\sim 1 \mathrm{Mb}$ away from the top SNP (DRGA0016155) identified for DLY pigs (Fig. 2g) [9].

\section{Suggestive QTL}

At the less strict thresholds for suggestive significance (Erhualian: 1/35974; Laiwu: 1/49343; and DLY: 1/56216), we identified an additional 76 QTL for the contents of 12 fatty acids in Erhualian, Laiwu and DLY pigs (see Additional file 2: Table S1). On average, two suggestive QTL were detected for each fatty acid in each population. Dissecting the molecular basis of these QTL with small effects is still a big challenge. However, these QTL regions harbor markers that can be potentially used in

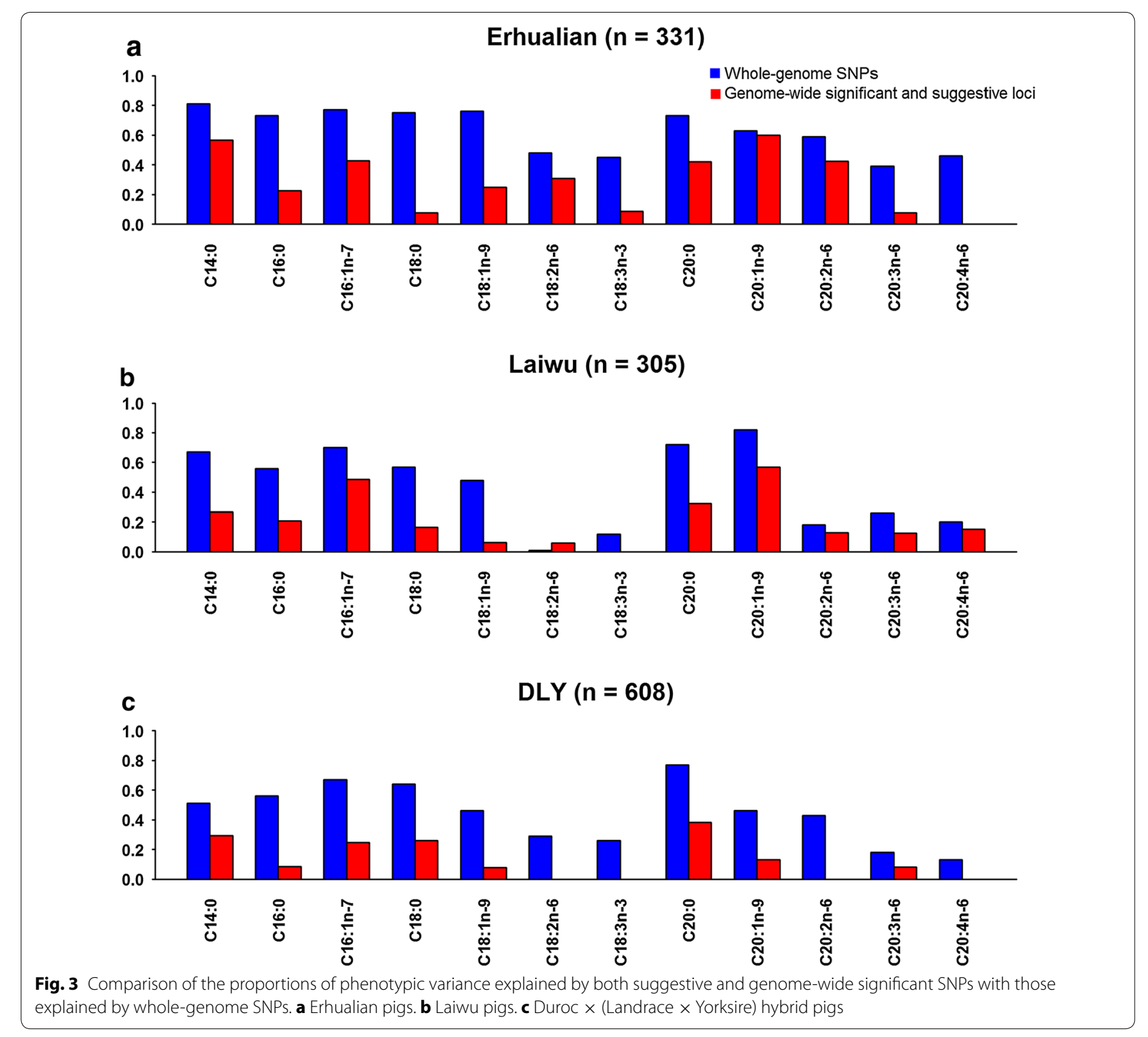


breeding schemes to improve fatty acid composition of meat. We compared the percentage of phenotypic variance explained by all the QTL that surpassed the suggestive significance threshold with the percentage of phenotypic variance that is collectively explained by all SNPs i.e., the marker-based heritability (Fig. 3). We found that the identified QTL explained more than $30 \%$ of the heritability or $20 \%$ of the phenotypic variance for most fatty acids, including C14:0, C16:0, C16:1, C18:0, C18:1, C18:2, C20:0, C20:1 and C20:2, in at least one population. For instance, we identified seven suggestive QTL for C14:0 for Erhualian pigs. These QTL together explained $69.8 \%$ of the heritability and $56.5 \%$ of the phenotypic variance (Additional file 2: Table S1 and Fig. 3). These results indicated that a small number of QTL can account for a considerable proportion of the breeding values for most fatty acid contents, which suggests that genetic improvement for fatty acid composition and pork quality by marker-assisted selection using a small set of SNPs can be considered.

\section{Conclusions}

We investigated fatty acid composition in the longissimus dorsi muscle of 1244 pigs from three divergent populations. The correlation patterns of fatty acids revealed that the metabolic pathway of polyunsaturated fatty acids is likely different from those of saturated and monounsaturated fatty acids. Through GWAS and GWAS meta-analysis, we detected 26 genome-wide significant QTL on eight chromosomes for eight fatty acids. These QTL not only replicated previously reported QTL but also included several novel QTL, which displayed complex patterns of pleiotropic effects, as well as populationshared and population-specific effects. At six significant QTL, we highlighted candidate genes related to fatty acid metabolism near the top associated SNPs. We showed that a small number of SNPs can explain a considerable proportion of phenotypic variance in the content of most fatty acids for at least one population. This study advances our understanding of the genetic architecture of fatty acid composition in porcine muscle.

\section{Additional files}

Additional file 1: Figure S1. Manhattan plot for the GWAS meta-analysis for the content of 12 fatty acids in five populations, including Erhualian, Laiwu, DLY, Sutai and White Duroc $\times$ Erhualian $F_{2}$ intercross. The solid line represents the genome-wide threshold $(0.05 / 28025)$. The dashed line indicates the suggestive threshold (1/28025). Significant associations that surpass the genome-wide threshold are plotted in different colors on each chromosome. The fatty acid traits associated with the genome-wide significant loci are marked above the top SNPS.

Additional file 2: Table S1. Loci of suggestive significance identified by GWAS for fatty acid composition in Laiwu, Erhualian and DLY pig populations.

\section{Authors' contributions}

$\mathrm{LH}$ and JR conceived and designed the experiments; JR supervised the experiments and revised the manuscript; BY analyzed the data and wrote the manuscript; WZ measured the phenotype, analyzed the data and wrote part of the manuscript; JZ measured the phenotype and analyzed part of the data; $\mathrm{CL}$ measured the phenotype; JM, CC, HA, XX, LL and SX participated in the animal slaughtering and sample collection. All authors read and approved the final manuscript.

\section{Acknowledgements}

The work was funded by Natural Science Foundation of China (31525023, 31230069, 31201776 and 31200926). We thank all colleagues in our lab for their enrollment in the animal management, sample collection, and $60 \mathrm{~K}$ genotyping work.

\section{Competing interests}

The authors declare that they have no competing interests.

Received: 8 July 2015 Accepted: 11 January 2016

Published online: 21 January 2016

\section{References}

1. Rothschild MF, Ruvinsky A. The genetics of the pig. 2nd ed. Wallingford: CAB International; 2011.

2. Fats and fatty acids in human nutrition. Report of an expert consultation. FAO Food Nutr Pap. 2010;91:1-166

3. Webb EC, O'Neill HA. The animal fat paradox and meat quality. Meat Sci. 2008;80:28-36.

4. Borkman M, Storlien LH, Pan DA, Jenkins AB, Chisholm DJ, Campbell LV. The relation between insulin sensitivity and the fatty-acid composition of skeletal-muscle phospholipids. N Engl J Med. 1993;328:238-44.

5. McCall FC, Telukuntla KS, Karantalis V, Suncion VY, Heldman AW, Mushtaq $M$, et al. Myocardial infarction and intramyocardial injection models in swine. Nat Protoc. 2012;7:1479-96.

6. Fan B, Onteru SK, Du ZQ, Garrick DJ, Stalder KJ, Rothschild MF. Genomewide association study identifies loci for body composition and structural soundness traits in pigs. PLoS One. 2011;6:e14726.

7. Ramayo-Caldas Y, Mercadé A, Castello A, Yang B, Rodriguez C, Alves E, et al. Genome-wide association study for intramuscular fatty acid composition in an Iberian x Landrace cross. J Anim Sci. 2012;90:2883-93.

8. Ren J, Duan Y, Qiao R, Yao F, Zhang Z, Yang B, et al. A missense mutation in PPARD causes a major QTL effect on ear size in pigs. PLoS Genet. 2011;7:e1002043.

9. Yang B, Zhang W, Zhang Z, Fan Y, Xie X, Ai H, et al. Genome-wide association analyses for fatty acid composition in porcine muscle and abdominal fat tissues. PLoS One. 2013;8:e65554.

10. Corominas J, Ramayo-Caldas Y, Puig-Oliveras A, Perez-Montarelo D, Noguera JL, Folch JM, et al. Polymorphism in the ELOVL6 gene is associated with a major QTL effect on fatty acid composition in pigs. PLoS One. 2013;8:e53687.

11. China National Commission of Animal Genetic Resources. Animal genetic resources in China pigs. Beijing: China Agricultural Press; 2011.

12. Folch J, Lees M, Sloane Stanley GH. A simple method for the isolation and purification of total lipides from animal tissues. J Biol Chem. 1957;226:497-509.

13. Ramos AM, Crooijmans RP, Affara NA, Amaral AJ, Archibald AL, Beever $\mathrm{JE}$, et al. Design of a high density SNP genotyping assay in the pig using SNPs identified and characterized by next generation sequencing technology. PLoS One. 2009;4:e6524.

14. Purcell $S$, Neale B, Todd-Brown $K$, Thomas L, Ferreira MA, Bender D, et al. PLINK: a tool set for whole-genome association and population-based linkage analyses. Am J Hum Genet. 2007;81:559-75.

15. Aulchenko YS, Ripke S, Isaacs A, van Duijn CM. GenABEL: an R library for genome-wide association analysis. Bioinformatics. 2007;23:1294-6.

16. Zhou X, Stephens M. Genome-wide efficient mixed-model analysis for association studies. Nat Genet. 2012;44:821-4. 
17. Segura V, Vilhjalmsson BJ, Platt A, Korte A, Seren U, Long Q, et al. An efficient multi-locus mixed-model approach for genome-wide association studies in structured populations. Nat Genet. 2012;44:825-30.

18. Willer CJ, Li Y, Abecasis GR. METAL: fast and efficient meta-analysis of genomewide association scans. Bioinformatics. 2010;26:2190-1.

19. Ai H, Huang L, Ren J. Genetic diversity, linkage disequilibrium and selec tion signatures in chinese and Western pigs revealed by genome-wide SNP markers. PLoS One. 2013;8:e56001.

20. Vance DE, Vance JE. Biochemistry of lipids, lipoproteins and membranes. 5th ed. Amsterdam: Elsevier; 2008.

21. Green CD, Ozguden-Akkoc CG, Wang Y, Jump DB, Olson LK. Role of fatty acid elongases in determination of de novo synthesized monounsaturated fatty acid species. J Lipid Res. 2010;51:1871-7.

22. Revilla M, Ramayo-Caldas Y, Castello A, Corominas J, Puig-Oliveras A, Ibanez-Escriche N, et al. New insight into the SSC8 genetic determination of fatty acid composition in pigs. Genet Sel Evol. 2014;46:28.

23. Jakobsson A, Westerberg R, Jacobsson A. Fatty acid elongases in mammals: their regulation and roles in metabolism. Prog Lipid Res. 2006;45:237-49.

24. Colbert CL, Kim CW, Moon YA, Henry L, Palnitkar M, McKean WB, et al. Crystal structure of Spot 14, a modulator of fatty acid synthesis. Proc Nat Acad Sci USA. 2010;107:18820-5.
25. Oh DY, Lee YS, la BM, Lee JY, Park YS, Lee JH, et al. Identification of exonic nucleotide variants of the thyroid hormone responsive protein gene associated with carcass traits and fatty acid composition in korean cattle. Asian-Australas J Anim Sci. 2014;27:1373-80.

26. Smith $\mathrm{S}$. The animal fatty acid synthase: one gene, one polypeptide, seven enzymes. FASEB J. 1994;8:1248-59.

27. Enoch HG, Catala A, Strittmatter P. Mechanism of rat liver microsomal stearyl-CoA desaturase. Studies of the substrate specificity, enzyme-substrate interactions, and the function of lipid. J Biol Chem. 1976;251:5095-103.

28. Estany J, Ros-Freixedes R, Tor M, Pena RN. A functional variant in the stearoyl-CoA desaturase gene promoter enhances fatty acid desaturation in pork. PLoS One. 2014;9:e86177.

29. Taniguchi M, Utsugi T, Oyama K, Mannen H, Kobayashi M, Tanabe Y, et al. Genotype of stearoyl-CoA desaturase is associated with fatty acid composition in Japanese Black cattle. Mamm Genome. 2004;15:142-8.

30. García-Fernández M, Gutiérrez-Gil B, García-Gámez E, Arranz J-J. Genetic variability of the Stearoyl-CoA desaturase gene in sheep. Mol Cell Probes. 2009;23:107-11.

\section{Submit your next manuscript to BioMed Central and take full advantage of:}

- Convenient online submission

- Thorough peer review

- No space constraints or color figure charges

- Immediate publication on acceptance

- Inclusion in PubMed, CAS, Scopus and Google Scholar

- Research which is freely available for redistribution

Submit your manuscript at

www.biomedcentral.com/submit

C Biomed Central 